\title{
THE
}

\section{Combining Smart Darting with Parallel Tempering Using Eckart Space: Application to Lennard-Jones Clusters}

\author{
Pablo Nigra \\ University of Rhode Island \\ David L. Freeman \\ University of Rhode Island, dfreeman@uri.edu \\ J. D. Doll
}

Follow this and additional works at: https://digitalcommons.uri.edu/chm_facpubs

Terms of Use

All rights reserved under copyright.

\section{Citation/Publisher Attribution}

Nigra, P., Freeman, D. L., \& Doll, J. D. (2005). Combining Smart Darting With Parallel Tempering Using Eckart Space: Applications to Lennard-Jones Clusters. Journal of Chemical Physics, 122(11), \#114113. doi: 10.1063/1.1858433

Available at: http://dx.doi.org/10.1063/1.1858433

This Article is brought to you for free and open access by the Chemistry at DigitalCommons@URI. It has been accepted for inclusion in Chemistry Faculty Publications by an authorized administrator of DigitalCommons@URI. For more information, please contact digitalcommons-group@uri.edu. 


\title{
Combining smart darting with parallel tempering using Eckart space: Application to Lennard-Jones clusters
}

\author{
Pablo Nigra ${ }^{\text {a) }}$ and David L. Freeman \\ Department of Chemistry, University of Rhode Island, Kingston, Rhode Island 02881 \\ J. D. Doll \\ Department of Chemistry, Brown University, Providence, Rhode Island 02912
}

(Received 20 October 2004; accepted 20 December 2004; published online 24 March 2005)

\begin{abstract}
The smart-darting algorithm is a Monte Carlo based simulation method used to overcome quasiergodicity problems associated with disconnected regions of configurations space separated by high energy barriers. As originally implemented, the smart-darting method works well for clusters at low temperatures with the angular momentum restricted to zero and where there are no transitions to permutational isomers. If the rotational motion of the clusters is unrestricted or if permutational isomerization becomes important, the acceptance probability of darting moves in the original implementation of the method becomes vanishingly small. In this work the smart-darting algorithm is combined with the parallel tempering method in a manner where both rotational motion and permutational isomerization events are important. To enable the combination of parallel tempering with smart darting so that the smart-darting moves have a reasonable acceptance probability, the original algorithm is modified by using a restricted space for the smart-darting moves. The restricted space uses a body-fixed coordinate system first introduced by Eckart, and moves in this Eckart space are coupled with local moves in the full $3 \mathrm{~N}$-dimensional space. The modified smart-darting method is applied to the calculation of the heat capacity of a seven-atom Lennard-Jones cluster. The smart-darting moves yield significant improvement in the statistical fluctuations of the calculated heat capacity in the region of temperatures where the system isomerizes. When the modified smart-darting algorithm is combined with parallel tempering, the statistical fluctuations of the heat capacity of a seven-atom Lennard-Jones cluster using the combined method are smaller than parallel tempering when used alone. (C) 2005 American Institute of Physics.
\end{abstract}

[DOI: $10.1063 / 1.1858433$ ]

\section{INTRODUCTION}

Small clusters of atoms and molecules have received much attention in recent years ${ }^{1}$ owing to their central role in such diverse areas as homogeneous nucleation and heterogeneous catalysis. In addition to their importance, the physical properties of the clusters themselves are inherently interesting, especially when contrasted with the properties of corresponding bulk materials. An important example of such correspondence is the phenomenon of phase change ${ }^{2}$ where clusters undergo rapid changes in physical properties with respect to their energy in a way that is reminiscent of bulk phase transitions. To study these important and interesting phase change regions, many computational methods have been either developed or used in ways that have proved to be generically important to the simulation community. Important examples of computational methods that have either partially or entirely evolved from the study of the phase change regions in clusters include $J$ walking, ${ }^{3}$ parallel tempering, ${ }^{4-6}$ smart darting, ${ }^{7}$ and applications of Tsallis statistics. ${ }^{8}$ The parallel tempering method has proved to be particularly powerful in overcoming quasiergodicity difficulties in the phase

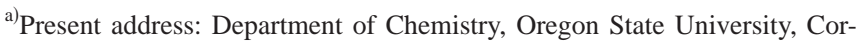
vallis, OR 97331.
}

change region as evidenced by the application of the parallel tempering algorithm to the study of the temperature dependent heat capacity of 38-atom Lennard-Jones clusters $\left(\mathrm{LJ}_{38}\right)$ in both the canonical ${ }^{9}$ and microcanonical ensembles. ${ }^{10}$ The complexity of the double-funneled potential energy surface in this system ${ }^{11}$ had defied previous simulation attempts principally owing to the difficulties in sampling both the icosahedral and cuboctahedral basins with the proper frequencies. With parallel tempering, the determination of the correct heat capacity has proved possible albeit with a large number of sampling points. Both the melting and solid-solid phase change regions can be resolved in $\mathrm{LJ}_{38}$ with parallel tempering methods. Recent studies using parallel tempering to examine the properties of mixed clusters ${ }^{12,13}$ have also illustrated the power of the approach.

Because of the success of the parallel tempering method in resolving details of the phase change regions in $\mathrm{LJ}_{38}$, it is natural to attempt to apply the method to even more complex problems. A good candidate is $\mathrm{LJ}_{75}$ (Ref. 14) which has a double-funnel potential surface much like $\mathrm{LJ}_{38}$ but with a transition state barrier about twice that found in $\mathrm{LJ}_{38}$. Our attempts to simulate the properties of $\mathrm{LJ}_{75}$ with parallel tempering have been unsuccessful. Although we have been able to find both basins in low temperature simulations of the system using parallel tempering, the results of our simula- 
tions have not been reproducible, probably because the transitions between the two primary basins in the system have not occurred at the correct frequency as dictated by the Boltzmann weight and phase space volumes. The purpose of the current work is to set the foundation for exploring an alternative sampling approach that has the potential for solving complex problems of which $\mathrm{LJ}_{75}$ is representative.

There have been a series of approaches designed to improve the performance of Monte Carlo based methods that combine sampling strategies. For example, Xu and Berne ${ }^{15}$ have combined $J$ walking and multicanonical based approaches, and separately Calvo and Doye ${ }^{16}$ have combined parallel tempering with multicanonical methods. Both studies have shown improvements in the performance of the combined methods when compared with the performance of multicanonical, $J$ walking, or parallel tempering when applied in isolation. In the current work we examine the smart darting method ${ }^{7}$ which we believe has the potential to enhance parallel tempering simulations in cases where parallel tempering is not sufficient. Our motivation for this particular choice comes from what we believe to be the shortcomings of parallel tempering in the simulation of $\mathrm{LJ}_{75}$. As we have indicated, we have found that a parallel tempering simulation of $\mathrm{LJ}_{75}$ does manage to find the two important and known basins of the potential energy surface, but over the length of simulations accessible by currently available computer resources, the frequency that the basins are accessed has fluctuations that are too large to enable reproducible results. Smart darting has virtues that can be expected to overcome this problem of ensuring transitions between basins with the proper frequency.

Smart darting has been formulated as a modification of the smart-walking method. ${ }^{17}$ Like some approaches developed to determine free-energy differences and transition theory rate constants, ${ }^{18}$ in smart darting a set of "dart" vectors is constructed that connect directly all the minima or sets of minima on the potential energy surface. In a pure smart darting calculation, Metropolis Monte Carlo simulations ${ }^{19}$ are enhanced with some predefined probability by transforming a current configuration to a new configuration by the addition of one of the constructed dart vectors. These darting moves enable efficient sampling of the disconnected basins on the potential energy surface. Darting moves are accepted or rejected in such a fashion that detailed balance is satisfied.

In the original implementation of smart darting ${ }^{7}$ the set of dart vectors is fixed in configuration space and chosen so that specific particles in specific orientations in one potential energy minimum connect specific particles in another potential energy minimum again with a specific orientation. Darting moves using such predefined vectors have a reasonable probability of acceptance provided the clusters do not rotate and provided permutational isomerization does not occur. If dart vectors are applied to configurations of atoms that have rotated or where the particle indices have effectively been permuted, the probability that a darting move is accepted becomes too small to modify the efficiency of ordinary Metropolis Monte Carlo simulations. To ensure reasonable acceptance of the darting moves, the original implementation is useful for systems having their angular momenta constrained to zero at temperatures where particle exchanges do not occur over the time scale of the simulation.

In this work we aim for an effective combination of smart darting with parallel tempering. In parallel tempering configurations from high temperature simulations are exchanged with simulations at lower temperatures. These high temperature structures have permuted configurations even if the angular momentum is constrained to zero. Because we want to include the effect of vibrational-rotational coupling in our simulations, we relax the angular momentum constraint used in Ref. 7 as well. Consequently, to have reasonable acceptance of the smart-darting moves, the original implementation requires modification.

In this work we demonstrate a useful modification of the smart-darting algorithm that allows the parallel tempering and smart-darting methods to be merged. Unlike the original algorithm, our modified approach permits the dart vectors to act on any geometry and any permutational isomer of the configuration and allows reasonable acceptance of dart moves for configurations differing significantly from any of the geometries of the potential energy minima. We accomplish this modification by performing the Metropolis moves in the full configuration space, but performing the darting moves within a restricted space first introduced by Eckart ${ }^{20}$ to solve problems concerned with molecular vibrations. For the moves in the restricted space it is necessary to introduce a correction to the usual Boltzmann acceptance probability. The correction is a Jacobian originating from a coordinate transformation in the full configurational space. This "Eckart space" has been used previously in other contexts, ${ }^{21}$ but we believe our current application of Eckart space to be different. The Eckart space techniques developed in this work may prove to be useful in other contexts. For example, similar use of Eckart space might enable the extension of the methods in Ref. 18 from surface reactions to reactions in the gas phase where rotations and isomerization events can also be expected to be important.

In the following section of this paper, we present the theoretical developments including a necessary review of Eckhart space, and how we apply this restricted space to smart darting moves in the context of Monte Carlo simulations. In Sec. III we apply our approach to simulate the properties of a seven-atom Lennard-Jones cluster and compare the efficiencies of both Metropolis and parallel tempering methods with and without smart darting. We summarize our conclusions in Sec. IV and propose future directions for the method.

\section{THEORY}

In this section we develop the theoretical tools needed to modify the original smart darting algorithm ${ }^{7}$ so that the angular momentum constraints can be removed. To accomplish this objective, we begin by introducing the notion of Eckart space. While various pieces of the development in Sec. II A have been formulated elsewhere, ${ }^{21}$ we find the review and organization to be essential in order to establish notation and 
make the subsequent sections clear. Following our discussion of Eckart space, we explain how we use Eckart space in the context of smart darting.

\section{A. Eckart space}

We consider a system of $N$ particles each having mass $m_{i}, i=1,2, \ldots, N$ described by the $N$ position vectors $\mathbf{r}_{i}$ in physical space $\mathbb{R}^{3}$ relative to an inertial frame. ${ }^{22-24}$ The reference frames used in the present work are right-handed systems. We use the notation $\left\{\mathbf{r}_{i}\right\}$ to represent the entire set of $N$ position vectors. It is convenient to use mass-weighted position vectors $\left(\mathbf{r}_{i} \rightarrow \sqrt{m_{i}} \mathbf{r}_{i}\right)$. Because the potential energy is invariant under translations, we fix the center of mass at the origin of the inertial frame, eliminating three degrees of freedom. As a result, we need only the first $(N-1)$ position vectors in the set $\left\{\mathbf{r}_{i}\right\}$ to specify a configuration; $\mathbf{r}_{N}$ can be obtained from the center of mass condition,

$$
\sum_{i=1}^{N} \sqrt{m_{i}} \mathbf{r}_{i}=0
$$

In addition to the inertial frame defined in the previous paragraph, we can also use a body-fixed frame to describe the location of an $N$-particle system, where the origin of the body-fixed frame is placed at the center of mass. For a particular configuration, the position vector $\mathbf{r}_{i}$ of particle $i$ is seen as either a rotating vector or a constant vector depending on the frame used to express the vector. The three Euler angles $\left\{\theta_{i}\right\}$ are used to determine the position of the rotating frame relative to the inertial frame. To describe any configuration in the body-fixed frame, we must specify fewer coordinates than those given by the first $(N-1)$ position vectors in $\left\{\mathbf{r}_{i}\right\}$. In other words, more than three Cartesian components are redundant now in $\left\{\mathbf{r}_{i}\right\}$, because a second condition arises that removes three rotational degrees of freedom. C. Eckart $^{20}$ has proposed a particular form for that condition. The Eckart approach begins by specifying a set of coordinates $\left\{\tilde{\mathbf{r}}_{i}\right\}$ that we call the reference configuration. Then any instantaneous configuration $\left\{\mathbf{r}_{i}\right\}$ is described relative to the reference by the relation,

$$
\sum_{i=1}^{N} \widetilde{\mathbf{r}}_{i} \times \mathbf{r}_{i}=0
$$

which is satisfied in both the inertial and body-fixed reference frames. Equation (2) is called the Eckart condition and plays a key role in the theory of molecular vibrations, where the reference configuration is taken to be the coordinates of some minimum of the potential energy. ${ }^{23,24}$ In fact, the reference configuration can be chosen arbitrarily (however, see Theorems II.2 and II.3 and the discussion that follows Theorem II.3). A more detailed explanation of the Eckart condition can be found in Ref. 21. The Eckart condition introduces three linear relations that remove three degrees of freedom. Consequently, a total of $(3 N-6)$ degrees of freedom are necessary to specify any configuration of the system. Those $(3 N-6)$ degrees of freedom constitute a vector space $\mathbb{R}^{3 N-6}$ that we call Eckart space.
In describing the current state of some $N$-particle system, it is important to distinguish configurations that represent different structures. To that end, we define two configurations $\alpha$ and $\beta$ to be equivalent in $\mathbb{R}^{3}$ if they can be superimposed by a proper rotation around the center of mass. In other words, $\alpha$ and $\beta$ are equivalent if there exits some rotation matrix $\mathbf{R}$ defined by a particular set of three Euler angles $\left\{\theta_{i}\right\}$ such that

$$
\mathbf{r}_{\beta, i}=\mathbf{R r}_{\alpha, i} \text {. }
$$

Sets of equivalent instantaneous configuration of a system in $\mathrm{R}^{3}$ can be identically mapped onto one or more $(3 N-6)$-dimensional vectors in Eckart space. As stated previously, we choose some reference configuration with a particular orientation in $\mathbb{R}^{3}$. To develop the expressions for the rotations needed to bring some instantaneous configuration to the orientations that comply with the Eckart condition [Eq. (2)], we define the auxiliary function

$$
\mathcal{L}\left(\left\{\mathbf{r}_{i}\right\}\right)=\sum_{i=1}^{N}\left(\mathbf{r}_{i}-\widetilde{\mathbf{r}}_{i}\right)^{2},
$$

where $\mathcal{L}$ is clearly the sum of the squares of the differences between the coordinates of the instantaneous configuration and the reference configuration. Because $\mathcal{L}$ is a function only of the Euler angles $\left\{\theta_{i}\right\}, \mathcal{L}$ is represented by a bounded threedimensional surface. We now prove a theorem that enables us to ensure the Eckart condition is satisfied.

Theorem II.1. Given an instantaneous configuration for some $\mathrm{N}$-particle system and a defined reference configuration in $R^{3}$, the Eckart condition is fulfilled at the extrema of $\mathcal{L}\left(\left\{\mathbf{r}_{i}\right\}\right)$.

Proof. We begin with the observation that $\mathbf{r}_{i}=\mathbf{r}_{i}\left(\theta_{j}\right)$. Differentiating,

$$
\frac{\partial \mathcal{L}\left(\left\{\mathbf{r}_{i}\right\}\right)}{\partial \theta_{j}}=-2 \sum_{i=1}^{N} \frac{\partial \mathbf{r}_{i}}{\partial \theta_{j}} \cdot \widetilde{\mathbf{r}}_{i}=2 \widetilde{\mathbf{n}}_{j} \cdot\left(\sum_{i=1}^{N} \widetilde{\mathbf{r}}_{i} \times \mathbf{r}_{i}\right) \forall j,
$$

where we have used the identity ${ }^{22} \partial \mathbf{r}_{i} / \partial \theta_{j}=\widetilde{\mathbf{n}}_{j} \times \mathbf{r}_{i}$ with $\widetilde{\mathbf{n}}_{j}$ a unit vector along the rotation axis of $\theta_{j}$. The directions of the three rotation axes of the $\left\{\theta_{j}\right\}$ depend on the arbitrary choice of orientation of the Cartesian frame. For the derivatives to be zero, the vector in the parenthesis of Eq. (5) must be the null vector, and the condition expressed in Eq. (2) is satisfied.

Theorem II.1 implies that there exist as many solutions to the Eckart condition as extrema in $\mathcal{L}\left(\left\{\mathbf{r}_{i}\right\}\right)$. We next establish the specific number of solutions to the Eckart condition by using the extrema of $\mathcal{L}\left(\left\{\mathbf{r}_{i}\right\}\right)$.

Theorem II.2. Given an instantaneous configuration for an $N$-particle system and a nonlinear reference configuration in $\mathbb{R}^{3}$, the Eckart condition admits four solutions at most.

Proof. To prove this theorem, it is convenient to rewrite Eq. (4) so that the terms not dependent on the Euler angles are eliminated. Taking $\mathbf{r}_{i}^{*}$ to be a particular coordinate vector, when we expand

$$
\left(\mathbf{r}_{i}^{*}-\widetilde{\mathbf{r}}_{i}\right)^{2}=\mathbf{r}_{i}^{* 2}+\widetilde{\mathbf{r}}_{i}^{2}-2 \mathbf{r}_{i}^{*} \cdot \widetilde{\mathbf{r}}_{i},
$$

we see that only the last term depends on the Euler angles. Consequently, the function $\mathcal{G}$ defined by the equation 


$$
\mathcal{G}\left(\left\{\mathbf{r}_{i}^{*}\right\}\right)=-\sum_{i=1}^{N} \mathbf{r}_{i}^{*} \cdot \widetilde{\mathbf{r}}_{i}
$$

has extrema with Euler angles coincident with the Euler angles at the extrema of $\mathcal{L}\left(\left\{\mathbf{r}_{i}^{*}\right\}\right)$. For that reason, we can now focus on $\mathcal{G}\left(\left\{\mathbf{r}_{i}^{*}\right\}\right)$. Next, we write $\mathbf{r}_{i}^{*}=\mathbf{R} \mathbf{r}_{i}$, where the $\mathbf{r}_{i}$ represent some initial orientation, and introduce this expression into Eq. (7),

$$
\mathcal{G}\left(\left\{\mathbf{R} \mathbf{r}_{i}\right\}\right)=-\sum_{i=1}^{N}\left(\mathbf{R} \mathbf{r}_{i}\right) \cdot \widetilde{\mathbf{r}}_{i},
$$

where the dependence on the rotation matrix $\mathbf{R}$ is explicit. We next express Eq. (8) in terms of quaternions ${ }^{22}$ rather than Euler angles. The quaternions are related by ${ }^{22}$

$$
\sum_{i=1}^{4} e_{i}^{2}=1
$$

and the rotation matrix expressed in terms of the quaternions is given by

$$
\begin{aligned}
& \mathbf{R} \\
& =\left[\begin{array}{ccc}
e_{1}^{2}+e_{2}^{2}-e_{3}^{2}-e_{4}^{2} & 2\left(e_{2} e_{3}+e_{1} e_{4}\right) & 2\left(e_{2} e_{4}-e_{1} e_{3}\right) \\
2\left(e_{2} e_{3}-e_{1} e_{4}\right) & e_{1}^{2}-e_{2}^{2}+e_{3}^{2}-e_{4}^{2} & 2\left(e_{3} e_{4}+e_{1} e_{2}\right) \\
2\left(e_{2} e_{4}+e_{1} e_{3}\right) & 2\left(e_{3} e_{4}-e_{1} e_{2}\right) & e_{1}^{2}-e_{2}^{2}-e_{3}^{2}+e_{4}^{2}
\end{array}\right] .
\end{aligned}
$$

Introducing Eq. (10) into Eq. (8) we obtain

$$
\begin{aligned}
\mathcal{G}\left(\left\{e_{i}\right\}\right)= & \sum_{i=1}^{N}\left\{e_{1}^{2}\left(-x_{i} \widetilde{x}_{i}-y_{i} \tilde{y}_{i}-z_{i} \widetilde{z}_{i}\right)+e_{2}^{2}\left(-x_{i} \widetilde{x}_{i}+y_{i} \widetilde{y}_{i}\right.\right. \\
& \left.+z_{i} \widetilde{z}_{i}\right)+e_{3}^{2}\left(x_{i} \widetilde{x}_{i}-y_{i} \widetilde{y}_{i}+z_{i} \widetilde{z}_{i}\right)+e_{4}^{2}\left(x_{i} \widetilde{x}_{i}+y_{i} \widetilde{y}_{i}\right. \\
& \left.-z_{i} \widetilde{z}_{i}\right)+2 e_{1} e_{2}\left(z_{i} \tilde{y}_{i}-y_{i} \widetilde{z}_{i}\right)+2 e_{1} e_{3}\left(x_{i} \widetilde{z}_{i}-z_{i} \widetilde{x}_{i}\right) \\
& +2 e_{1} e_{4}\left(y_{i} \widetilde{x}_{i}-x_{i} \widetilde{y}_{i}\right)+2 e_{2} e_{3}\left(-x_{i} \widetilde{y}_{i}-y_{i} \widetilde{x}_{i}\right) \\
& \left.+2 e_{2} e_{4}\left(-x_{i} \widetilde{z_{i}}-z_{i} \widetilde{x}_{i}\right)+2 e_{3} e_{4}\left(-y_{i} \widetilde{z}_{i}-z_{i} \tilde{y}_{i}\right)\right\} .
\end{aligned}
$$

Noting that $\mathcal{G}$ is a quadratic form in the $\left\{e_{i}\right\}$, we can write

$$
\mathcal{G}\left(\left\{e_{i}\right\}\right)=\sum_{l=1}^{4} \sum_{k=1}^{4} P_{l, k} e_{l} e_{k},
$$

with $\mathbf{P}$ a $4 \times 4$ symmetric matrix whose elements are given by

$$
\begin{aligned}
& P_{1,1}=-\sum_{i=1}^{N}\left(x_{i} \tilde{x}_{i}+y_{i} \tilde{y}_{i}+z_{i} \tilde{z}_{i}\right), \\
& P_{1,2}=\sum_{i=1}^{N}\left(z_{i} \tilde{y}_{i}-y_{i} \tilde{z}_{i}\right), \\
& P_{1,3}=\sum_{i=1}^{N}\left(x_{i} \tilde{z}_{i}-z_{i} \tilde{x}_{i}\right), \\
& P_{1,4}=\sum_{i=1}^{N}\left(y_{i} \tilde{x}_{i}-x_{i} \tilde{y}_{i}\right),
\end{aligned}
$$

$$
\begin{aligned}
& P_{2,2}=\sum_{i=1}^{N}\left(-x_{i} \widetilde{x}_{i}+y_{i} \tilde{y}_{i}+z_{i} \tilde{z}_{i}\right), \\
& P_{2,3}=-\sum_{i=1}^{N}\left(x_{i} \tilde{y}_{i}+y_{i} \widetilde{x}_{i}\right), \\
& P_{2,4}=-\sum_{i=1}^{N}\left(x_{i} \widetilde{z}_{i}+z_{i} \tilde{x}_{i}\right), \\
& P_{3,3}=\sum_{i=1}^{N}\left(x_{i} \tilde{x}_{i}-y_{i} \tilde{y}_{i}+z_{i} \widetilde{z}_{i}\right), \\
& P_{3,4}=-\sum_{i=1}^{N}\left(y_{i} \tilde{z}_{i}+z_{i} \tilde{y}_{i}\right), \\
& P_{4,4}=\sum_{i=1}^{N}\left(x_{i} \tilde{x}_{i}+y_{i} \tilde{y}_{i}-z_{i} \tilde{z}_{i}\right) .
\end{aligned}
$$

Equation (12), together with Eq. (9), demonstrate that the problem of finding the extrema of $\mathcal{G}\left(\left\{e_{i}\right\}\right)$ is a constrained extrema problem. Using the method of Lagrange multipliers, the extrema of $\mathcal{G}\left(\left\{e_{i}\right\}\right)$ occur when the first derivatives of the function

$$
\mathcal{F}\left(\left\{e_{i}\right\}\right)=\mathcal{G}\left(\left\{e_{i}\right\}\right)-\lambda \sum_{l=1}^{4} e_{l}^{2}
$$

vanish, with $\lambda$ being a Lagrange multiplier. Then,

$$
\frac{\partial \mathcal{F}}{\partial e_{i}}=2 \sum_{j=1}^{4} P_{i, j} e_{j}-2 \lambda e_{i} \quad \forall i,
$$

which are zero when

$$
\sum_{j=1}^{4} P_{i, j} e_{j}^{*}=\lambda e_{i}^{*},
$$

where the superscript $*$ on the $e_{i}$ denotes the location of the extrema. Equation (16) is a familiar eigenvalue problem that in matrix form is given by

$$
(\mathbf{P}-\lambda \mathbf{I}) \mathbf{e}^{*}=0 \text {. }
$$

Because $\mathbf{P}$ is a $4 \times 4$ symmetric matrix, the Lagrange multiplier $\lambda$ can be any of the four real eigenvalues of $\mathbf{P}$. Each of the four eigenvectors $\mathbf{e}_{j}^{*}$ of $\mathbf{P}$ contains four quaternions. We use those $\left\{e_{i, j}^{*}\right\}$ to build the rotation matrices [Eq. (10)] that bring the initial configuration to the orientations where $\mathcal{G}$ attains its extrema. Finally, we conclude that $\mathcal{G}$ (and by the coincidence of extrema, $\mathcal{L}$ ) must attain four extrema at most. From Theorem II.1, it is evident that the Eckart condition admits four solutions at most.

It is useful for further analysis to examine the nature of the extrema of $\mathcal{G}\left(\left\{e_{i}\right\}\right)$ (or equivalently $\mathcal{L}$ ). We first consider $\lambda_{1} \leqslant \lambda_{2} \leqslant \lambda_{3} \leqslant \lambda_{4}$, and introduce Eq. (16) into Eq. (12) to obtain 


$$
\mathcal{G}\left(\left\{e_{\alpha, i}^{*}\right\}\right)=\lambda_{\alpha},
$$

which shows that $\mathcal{G}\left(\left\{e_{i}\right\}\right)$ attains a minimum $\left(\mathcal{G}\left(\left\{e_{1, i}^{*}\right\}\right)=\lambda_{1}\right)$ and a maximum $\left(\mathcal{G}\left(\left\{e_{4, i}^{*}\right\}\right)=\lambda_{4}\right)$. If there is no degeneracy, the intermediate values of $\lambda$ represent saddle points. ${ }^{25}$ The matrix $\mathbf{P}$ of Eq. (13) has trace equal to zero, showing, that $\mathbf{P}$ has both positive and negative eigenvalues. The first element of $\mathbf{P}, P_{1,1}$, equals $\mathcal{G}\left(\left\{\mathbf{r}_{i}\right\}\right)$ [see Eq. (7)], and the last three elements of the first row are the Cartesian components of the vector function,

$$
\mathcal{E}\left(\left\{\mathbf{r}_{i}\right\}\right)=\sum_{i=1}^{N} \widetilde{\mathbf{r}}_{i} \times \mathbf{r}_{i},
$$

whose zeros define the Eckart condition. When a configuration is oriented so that it satisfies the Eckart condition, $\mathbf{P}$ becomes block diagonal,

$$
\mathbf{P}^{\alpha}=\left[\begin{array}{cccc}
\lambda_{\alpha} & 0 & 0 & 0 \\
0 & P_{2,2} & P_{2,3} & P_{2,4} \\
0 & P_{3,2} & P_{3,3} & P_{3,4} \\
0 & P_{4,2} & P_{4,3} & P_{4,4}
\end{array}\right] .
$$

From the expression for $P_{1,1}$ in Eq. (13) along with Eq. (20), it is evident that we can write

$$
\lambda_{\alpha}=-\sum_{i=1}^{N} \mathbf{r}_{i} \cdot \widetilde{\mathbf{r}}_{i}
$$

We can understand the meaning of the lowest and highest eigenvalues of $\mathbf{P}$ geometrically. Each value of $\lambda$ represents a different orientation of the configuration of the system compared to the reference structure. The orientation of the smallest eigenvalue $\lambda_{1}$ corresponds to the smallest leastsquares difference between the coordinates of the reference and the system configuration [See Eq. (4)]. In the leastsquares sense, the orientation associated with $\lambda_{1}$ is the "best match" between the current configuration and the reference configuration. In a similar manner, the orientation associated with the largest eigenvalue $\lambda_{4}$ corresponds to the "worst match" between the current configuration and the reference configuration.

As is discussed later in this paper, during a Monte Carlo simulation, to make a move in Eckart space with some frequency $f$, the instantaneous configuration generated by an ordinary move in the previous step can be introduced into the Eckart subspace by choosing an eigenvector of $\mathbf{P}$. This key idea is used in the development of the modified smart darting algorithm.

The results of Theorem II.2 can be used to analyze the case of a linear reference in $\mathbb{R}^{3}$.

Theorem II.3. Given a configuration for an $N$-particle system and a linear reference in $R^{3}$, the Eckart condition admits an infinite number of solutions if the configuration is nonlinear with $N>2$. For a linear configuration with $N \geqslant 2$, the Eckart condition admits only two solutions.

Proof. For simplicity, we let the linear reference lie along the $x$ axis so that the coordinates of each particle $i$ are $\left\{\widetilde{x}_{i}, 0,0\right\}$. For a configuration oriented to fulfill the Eckart condition, it can be readily seen from Eq. (13) and Eq. (20) that $\mathbf{P}$ is diagonal,

$$
\mathbf{P}=\left[\begin{array}{cccc}
-\lambda & 0 & 0 & 0 \\
0 & -\lambda & 0 & 0 \\
0 & 0 & \lambda & 0 \\
0 & 0 & 0 & \lambda
\end{array}\right],
$$

with

$$
\lambda=\sum_{i=1}^{N} x_{i} \widetilde{x}_{i}
$$

Equation (22) shows that $\mathrm{P}$ has only two different eigenvalues when the reference is linear. From Eq. (23), we infer that $\mathcal{G}$ attains its extrema along the axis of the reference, which we have taken to be the $x$ axis. From a geometric point of view, when the Eckart condition is satisfied, the configuration is in alignment according to the type of the extremum. For a nonlinear configuration with $N>2$, however, each extremum of $\mathcal{G}$ corresponds to infinite set of orientations of the configuration around the axis of the reference. In other words, there are infinite sets of the $\left\{\mathbf{r}_{i}\right\}$ consistent with each type of alignment. We can conclude that there exist an infinite number of solutions to the Eckart condition. For a linear configuration with $N \geqslant 2$, there is one set of $\left\{\mathbf{r}_{i}\right\}$, namely, $\left\{x_{i}, 0,0\right\}$, consistent with each extremum. Consequently, there exist only two solutions to the Eckart condition.

Theorems II.2 and II.3 state that more than one element of Eckart space is related to a given configuration. Because it is easier to choose among four elements than to choose among an infinite set of elements, it is best to avoid selecting linear configurations when defining reference configurations.

It is important to recognize that each vector in Eckart space is characterized both by a configuration of particles as well as by an eigenvalue $\lambda_{\alpha}$ obtained using Eq. (17). When a nonlinear reference is used, four equivalent configurations are associated with four vectors labeled with different values of $\lambda_{\alpha}$. We find it convenient to divide Eckart space $\mathcal{S}$ into four partitions each corresponding to the kind of eigenvalue $\lambda_{\alpha}$ using the notation,

$$
\mathcal{S}=\bigcup_{\alpha=1}^{4} \mathcal{S}^{(\alpha)}
$$

where $\mathcal{S}^{(1)}$ contains the vectors characterized by the smallest eigenvalues $\lambda_{1}$ obtained from Eq. (17), $\mathcal{S}^{(2)}$ contains the vectors characterized by the second smallest eigenvalues $\lambda_{2}$, and so on. It is clear that none of the vectors inside any of the partitions $\mathcal{S}^{(\alpha)}$ represent equivalent configurations. Finally, we remark that dividing Eckart space as in Eq. (24) does not imply that each of the partitions $\mathcal{S}^{(\alpha)}$ is a vector space. Eckart space $\mathcal{S}$ is a vector space because any linear combination of vectors in $\mathcal{S}$ results in another vector inside $\mathcal{S}$. In contrast, any linear combination of vectors in, for instance, $\mathcal{S}^{(1)}$ does not necessarily result in another vector in $\mathcal{S}^{(1)}$. 


\section{B. Smart darting in Eckart space: Distinguishable particles}

In this section, we introduce our implementation of the smart-darting algorithm for the special case of distinguishable particles. The extension of the approach to indistinguishable particles involves some additional complications that are discussed in the subsequent section. This section is organized so that each step in the algorithm is given with a discussion of the justification for that particular step.

We execute smart darting moves in Eckart space by restricting the possible moves to occur only within the region $\mathcal{S}^{(1)}$ defined in Sec. II A. We recall that a vector in $\mathcal{S}^{(1)}$ represents a configuration with associated matrix $\mathbf{P}^{1}$ [see Eq. (20)] whose first element is its smallest eigenvalue $\lambda_{1}$; i.e., such that the particles are rotated to give the best match to the reference configuration in the least-squares sense. Consequently, the vectors restricted to $\mathcal{S}^{(1)}$ represent different configurations for the case of distinguishable particles. Because we represent configurations by vectors in Eckart space, we need to make an important point about the choice of internal coordinates suitable for the current application. An element of Eckart space is usually represented by some suitable set of $(3 N-6)$ scalar internal coordinates $\left\{q_{i}\right\}$. The $\left\{q_{i}\right\}$ are defined so that they are invariant under rotations. In addition, the $\left\{q_{i}\right\}$ must always reflect the choice of reference. In that last respect, however, some of the commonly used $\left\{q_{i}\right\}$ in molecular physics may not be suitable coordinates for use in Eckart space. For example, the internal bond-angle coordinates, ${ }^{23,24}$ which are rotationally invariant, are defined to describe the shape of a configuration by specifying the values of internal bonds and angles, but not related to any reference. Obviously, more than one element (four for a nonlinear reference) of Eckart space is characterized by the same set of $(3 N-6)$ bond-angle coordinates. It is required that a one-to-one relation between the $\left\{q_{i}\right\}$ and the elements of Eckart space exist in order that the $\left\{q_{i}\right\}$ become suitable coordinates. A convenient set of $\left\{q_{i}\right\}$ for our computational purposes is the one obtained by directly picking $(3 N-6)$ Cartesian coordinates out of the initial $3 \mathrm{~N}$ Cartesian coordinates with the origin at the center of mass. The remaining six Cartesian coordinates can be then expressed as functions of the selected $(3 N-6)$ coordinates by using Eqs. (1) and (2). That is the approach we follow in the Appendix where we derive the neccessary Jacobian of transformation to work in Eckart space.

The algorithm we describe here is designed to be coupled with a local-move algorithm in which no restriction on degrees of freedom is applied. In the current work, for the local-move algorithm we take the usual Metropolis method and we apply parallel tempering or smart-darting moves with some predefined frequencies. In what follows, we make use of two kinds of vectors; the $N$ position vectors $\left\{\mathbf{r}_{i}\right\}$ and $(3 N-6)$-dimensional vectors $\boldsymbol{\Gamma}$ in Eckart space. As discussed in Sec. II A, the algorithm is facilitated by choosing a nonlinear configuration as the reference. In our approach we have chosen the lowest energy structure of the system as the reference configuration, but that particular choice is one of convenience and is not mandated by the method.
Prior to beginning the simulation, after choosing the reference configuration, we construct a set of $M$ minima of the potential energy surface to be used in the smart-darting algorithm. The set of $M$ minima can encompass all the possible minima on a potential surface or some conveniently chosen subset of the available minima. Using the reference configuration, the minima are located in Eckart space and defined by the Eckart vectors $\boldsymbol{\Gamma}_{i}, i=1,2, \ldots M$. Each $\boldsymbol{\Gamma}_{i}$ is constructed using the lowest eigenvalue of the corresponding $\mathbf{P}$ matrix. The approach used in bringing the configuration of each minimum into the Eckart space defined by our chosen reference configuration is identical to Step 1 listed below. In what follows, we refer to each $\boldsymbol{\Gamma}_{i}$ constructed from one of the $M$ minima as a template. In addition to the Eckart vectors defining the minima to be used in the simulation, we also construct a set of dart vectors in Eckart space defined by

$$
\boldsymbol{\Delta}_{i, j}=\boldsymbol{\Gamma}_{i}-\boldsymbol{\Gamma}_{j} .
$$

For distinguishable particles, the smart-darting portion of the algorithm consists of the steps that are as follows.

Step 1. We bring the instantaneous configuration $\left\{\mathbf{r}_{i}^{\prime}\right\}$ generated by the local-move algorithm into $\mathcal{S}^{(1)}$.

To accomplish Step 1, we first refer all the $\left\{\mathbf{r}_{i}^{\prime}\right\}$ to the center of mass. Next we form the matrix $\mathbf{P}$ of Eq. (13) and diagonalize it. We then take the eigenvector corresponding to the smallest eigenvalue and use it to construct the rotation matrix $\mathbf{R}$ of Eq. (10). Finally we rotate the $\left\{\mathbf{r}_{i}^{\prime}\right\}$ according to

$$
\mathbf{r}_{i}=\mathbf{R} \mathbf{r}_{i}^{\prime}
$$

It is clear that the instantaneous configurations can be characterized either by the vectors $\left\{\mathbf{r}_{i}\right\}$ or by a vector $\boldsymbol{\Gamma}$. As explained in Sec. II A, both kinds of vectors contain the $(3 N-6)$ coordinates needed to define the Eckart space. The $\left\{\mathbf{r}_{i}\right\}$ also contain the six redundant coordinates.

Step 2. We locate the template $\boldsymbol{\Gamma}_{i}$ in $\mathcal{S}^{(1)}$ that is closest to the instantaneous configuration $\boldsymbol{\Gamma}$.

To locate the closest template to the instantaneous configuration, we calculate the distances between $\boldsymbol{\Gamma}$ and all the $\boldsymbol{\Gamma}_{i}$. To determine these distances, we first specify the set of internal coordinates. Following the analysis given in the Appendix, we take the set of Cartesian coordinates $\left\{x_{N}, y_{N}, z_{N}, y_{N-1}, z_{N-1}, z_{N-2}\right\}$ to be the redundant coordinates. With this choice of redundant coordinates, the square of the distance is given by

$$
\begin{aligned}
d_{i}^{2}= & \left(\boldsymbol{\Gamma}-\boldsymbol{\Gamma}_{i}\right)^{2}=\left(x_{N-1}-x_{N-1, i}\right)^{2}+\left(x_{N-2}-x_{N-2, i}\right)^{2}+\left(y_{N-2}\right. \\
& \left.-y_{N-2, i}\right)^{2}+\sum_{k=1}^{N-3}\left(\mathbf{r}_{k}-\mathbf{r}_{k, i}\right)^{2} .
\end{aligned}
$$

Step 3. We choose a target template $\boldsymbol{\Gamma}_{j}$ with uniform probability $t_{j}=(M-1)^{-1}$ and construct a new configuration

$\boldsymbol{\Gamma}^{\prime}=\boldsymbol{\Gamma}+\boldsymbol{\Delta}_{j, i}$ 
Because the relation between each of the six redundant coordinates and the remaining $(3 N-6)$ coordinates is linear [see Eq. (2)], we can write any of the redundant primed coordinates as a simple linear sum of the unprimed coordinates. For example,

$$
z_{N}^{\prime}=z_{N}+z_{N, j}-z_{N, i}
$$

where $z_{N}, z_{N, i}$, and $z_{N, j}$ belong to the instantaneous configuration and the templates $i$ and $j$, respectively. Consequently, a convenient alternative to Eq. (28) for the new configuration is

$$
\mathbf{r}_{k}^{\prime}=\mathbf{r}_{k}+\mathbf{r}_{k, j}-\mathbf{r}_{k, i}
$$

with $k=1, \ldots, N$.

Step 4. We accept the new configuration $\boldsymbol{\Gamma}^{\prime}$ with probability

$$
A\left(\boldsymbol{\Gamma} \rightarrow \boldsymbol{\Gamma}^{\prime}\right)= \begin{cases}0 & \text { if } \boldsymbol{\Gamma}^{\prime} \notin \mathcal{S}^{(1)} \\ 0 & \text { if } \left.\left|\boldsymbol{\Gamma}^{\prime}-\boldsymbol{\Gamma}_{j}\right|>\left|\boldsymbol{\Gamma}^{\prime}-\boldsymbol{\Gamma}_{k}\right| \text { for some } k \neq j\right) \\ \min \left\{1, \frac{\rho^{\prime}}{\rho}\right\} & \text { (if } \left.\left|\boldsymbol{\Gamma}^{\prime}-\boldsymbol{\Gamma}_{j}\right|<\left|\boldsymbol{\Gamma}^{\prime}-\boldsymbol{\Gamma}_{k}\right| \forall k \neq j\right),\end{cases}
$$

where $\rho$ is the probability density.

In Sec. II A, we have emphasized that linear combinations of vectors in Eckart space are also Eckart space vectors, but the resulting vectors may or may not be elements of $\mathcal{S}^{(1)}$. To determine if a resultant $\boldsymbol{\Gamma}^{\prime}$ is an element of $\mathcal{S}^{(1)}$, we construct the matrix $\mathbf{P}$ for $\boldsymbol{\Gamma}^{\prime}$ using Eq. (13) and compare the first element of the constructed $\mathbf{P}, P_{1,1}$, with the smallest eigenvalue of $\mathbf{P}$. Agreement between $P_{1,1}$ and the lowest eigenvalue implies the resultant vector is an element of $\mathcal{S}^{(1)}$. To guarantee that the target template $\boldsymbol{\Gamma}_{j}$ is the closest template to $\boldsymbol{\Gamma}^{\prime}$, we use the procedure outlined in Step 2 to calculate the distances between $\Gamma^{\prime}$ and all the remaining templates. The probability density $\rho$ is expressed as a function of $(3 N-3)$ generalized coordinates given by the three Euler angles $\left\{\theta_{i}\right\}$, and the $(3 N-6)$ internal coordinates comprising the Eckart space vector $\boldsymbol{\Gamma}$. In the canonical ensenble, $\rho$ is simply the product of the Boltzmann factor and the Jacobian for the transformation into generalized coordinates [Eq. (A27)]

$$
\rho\left(\theta_{i}, \boldsymbol{\Gamma}\right)=\exp [-\beta U(\boldsymbol{\Gamma})] \sin \theta_{2}|\bar{J}(\boldsymbol{\Gamma}) / c|,
$$

where $\beta$ is the Boltzmann inverse temperature, $U$ is the potential energy, $\theta_{2}$ is the second Euler angle, $c$ is a constant, and $\bar{J}(\boldsymbol{\Gamma})$ is the part of the Jacobian that depends only on the internal coordinates. The expression for $\bar{J}(\boldsymbol{\Gamma})$ is given in Eq. (A28) of the Appendix. Finally, if $\sin \theta_{2}^{\prime}=\sin \theta_{2}($ See Step 5), the ratio of probability densities in Eq. (31) depends only on the internal coordinates of the initial and final configurations

$$
\frac{\rho^{\prime}}{\rho}=\exp \left[-\beta \Delta U\left(\boldsymbol{\Gamma}^{\prime}, \boldsymbol{\Gamma}\right)\right]\left|\frac{\bar{J}\left(\boldsymbol{\Gamma}^{\prime}\right)}{\bar{J}(\boldsymbol{\Gamma})}\right|,
$$

with $\Delta U\left(\boldsymbol{\Gamma}^{\prime}, \boldsymbol{\Gamma}\right)=U\left(\boldsymbol{\Gamma}^{\prime}\right)-U(\boldsymbol{\Gamma})$.

Step 5. If the new configuration $\boldsymbol{\Gamma}^{\prime}$ is rejected in Step 4, we keep the old configuration in its original orientation and return to local moves using the local-move algorithm. If the darted configuration $\boldsymbol{\Gamma}^{\prime}$ is accepted, we rotate the new configuration to the initial orientation of the instantaneous configuration.

The latter is the inverse of Step 1. In other words, we rotate the position vectors of the new configuration according to

$$
\mathbf{r}_{i}=\mathbf{R}^{T} \mathbf{r}_{i}^{\prime}
$$

where $\mathbf{R}^{T}$ is the transpose of $\mathbf{R}$ in Step 1 .

The five steps outlined above provide the details for the algorithm as applied to systems composed of distinguishable particles. It is now necessary to verify that the algorithm satisfies detailed balance. As discussed above, the process begins by identifying the template that is closest to the instantaneous configuration; i.e., Step 2 above. We then define a convenient, trial probability for darting moves from that host template. The probability of generating a configuration $\boldsymbol{\Gamma}^{\prime(j)}$ associated with a target template $\boldsymbol{\Gamma}_{j}$ from a configuration $\boldsymbol{\Gamma}^{(i)}$ associated with a host template $\boldsymbol{\Gamma}_{i}$ can be written as

$$
T\left(\boldsymbol{\Gamma}^{(i)} \rightarrow \boldsymbol{\Gamma}^{\prime(j)}\right)=t_{j, i} \delta\left(\boldsymbol{\Gamma}^{\prime(j)}-\left[\boldsymbol{\Gamma}^{(i)}+\boldsymbol{\Delta}_{j, i}\right]\right),
$$

where $t_{j, i}$ is the probability of choosing the dart $\boldsymbol{\Delta}_{j, i}$. The Dirac delta function $\delta\left(\boldsymbol{\Gamma}^{\prime(j)}-\left[\boldsymbol{\Gamma}^{(i)}+\boldsymbol{\Delta}_{j, i}\right]\right)$ gives the probability of forming $\boldsymbol{\Gamma}^{\prime(j)}$ on condition that $\boldsymbol{\Delta}_{j, i}$ has been chosen. Furthermore, the darts are chosen uniformly out of a set of $(M-1)$ darts so that

$$
t_{j, i}=1 /(M-1) \text {. }
$$

With the definition given in Eq. (35), the normalization of $T\left(\boldsymbol{\Gamma}^{(i)} \rightarrow \boldsymbol{\Gamma}^{\prime(j)}\right)$ must go first throughout the Eckart space, and then among the available darts

$$
\sum_{j=1}^{M-1} \int T\left(\boldsymbol{\Gamma}^{(i)} \rightarrow \boldsymbol{\Gamma}^{\prime(j)}\right) d \boldsymbol{\Gamma}^{\prime}=1 .
$$

Detailed balance is guaranteed by choosing the acceptance probability $A\left(\boldsymbol{\Gamma}^{(i)} \rightarrow \boldsymbol{\Gamma}^{\prime(j)}\right)$ to be 


$$
A\left(\boldsymbol{\Gamma}^{(i)} \rightarrow \boldsymbol{\Gamma}^{\prime(j)}\right)= \begin{cases}0 & \text { if } \boldsymbol{\Gamma}^{\prime(j)} \notin \mathcal{S}^{(1)} \\ 0 & \left(\text { if }\left|\boldsymbol{\Gamma}^{\prime(j)}-\boldsymbol{\Gamma}_{j}\right|>\left|\boldsymbol{\Gamma}^{\prime(j)}-\boldsymbol{\Gamma}_{k}\right| \text { for some } k \neq j\right) \\ \min \left\{1, \frac{T\left(\boldsymbol{\Gamma}^{\prime(j)} \rightarrow \boldsymbol{\Gamma}^{(i)}\right) \rho^{\prime}}{T\left(\boldsymbol{\Gamma}^{(i)} \rightarrow \boldsymbol{\Gamma}^{\prime(j)}\right) \rho}\right\} & \left(\text { if }\left|\boldsymbol{\Gamma}^{\prime(j)}-\boldsymbol{\Gamma}_{j}\right|<\left|\boldsymbol{\Gamma}^{\prime(j)}-\boldsymbol{\Gamma}_{k}\right| \forall k \neq j\right) .\end{cases}
$$

In the cases that either $\boldsymbol{\Gamma}^{(i)}$ or $\boldsymbol{\Gamma}^{\prime(j)}$ do not belong to $\mathcal{S}^{(1)}$, it is clear that detailed balance is fulfilled, because acceptance probability is zero for both forward and reverse moves. In the case that the target template $\boldsymbol{\Gamma}_{j}$ is not the closest template to $\Gamma^{(j)}$, the acceptance probability is zero. For the reverse move, the trial probability is zero, because no dart can return the system from $\Gamma^{\prime(j)}$ to $\Gamma^{(i)}$. Again, in this case detailed balance is clearly satisfied. If the acceptance and trial probabilities do not vanish for both forward and reverse moves, it is not difficult to verify that

$$
T\left(\boldsymbol{\Gamma}^{(i)} \rightarrow \boldsymbol{\Gamma}^{\prime(j)}\right)=T\left(\boldsymbol{\Gamma}^{\prime(j)} \rightarrow \boldsymbol{\Gamma}^{(i)}\right),
$$

so that the expression for the acceptance probability in Eq. (38) contains only the ratio of the probability densities; i.e., we obtain Eq. (31).

\section{Indistinguishable particles}

When the particles of the system are indistinguishable, there are additional complications that we now consider. Because of permutational symmetry a particular configuration is represented by $N$ ! vectors inside $\mathcal{S}^{(1)}$. Consequently, the distinguishable-particle smart-darting algorithm developed in Sec. II B is not directly applicable to the present case. For indistinguishable particles we replace $\mathcal{S}^{(1)}$ with a region of Eckart space where each of the $N$ ! indistinguishable permutational isomers is represented by only one vector $\boldsymbol{\Gamma}$. We require that such a region, labeled as $O^{(1)}$ be part of $\mathcal{S}^{(1)}$. Unlike $S^{(1)}$, a region $O^{(1)}$ cannot be defined in a unique manner. If $m$ represents the number of configurations of the system, the number of ways to define $O^{(1)}$ is $N !^{m}$, where $m$ is, in fact, infinite. However, there are ways of defining a reasonable region $O^{(1)}$ suitable for an effective smart-darting method. An intuitive image of a suitable shape for $O^{(1)}$ is provided by the following consideration. Given an instantaneous configuration that is a small deformation of a template $\boldsymbol{\Gamma}_{i}$, we want the vector $\boldsymbol{\Gamma}$ in $O^{(1)}$ to be (among the $N$ ! possibilities in $\mathcal{S}^{(1)}$ ) the one closest to $\boldsymbol{\Gamma}_{i}$ in the sense $\boldsymbol{\Gamma} \approx \boldsymbol{\Gamma}_{i}$. We can then expect a target configuration formed by darting to have an appreciable acceptance probability.

We have found the following algorithm of constructing $O^{(1)}$ to be an effective basis for smart darting in the applications studied in the current work. In the steps that follow we use Roman numerals to differentiate these steps from the algorithm introduced in Sec. II B. The steps given below are constructed to build a unique region $O^{(1)}$.

Step I. Select two particles of the reference configuration that are not collinear with the center of mass and that possess some distinct attributes.
In the current work, we choose the particle that is closest to the center of mass and the particle that is farthest from the center of mass. This step is executed before the simulation is started.

Step II. Select two particles in the instantaneous configuration that possess the same attributes as those in Step I.

For Step II, it does not matter if the chosen particles are collinear with the center of mass.

Step III. Match the two selected particles of the instantaneous configuration to those of the reference and rotate the instantaneous configuration so that the metric distance between the instantaneous configuration and the reference for the matched particles is a minimum.

In this step, we construct $\mathbf{P}$ and diagonalize $\mathbf{P}$ [Eq. (13)] using only the matched pairs (closest-closest, farthestfarthest). We then take the eigenvector belonging to the smallest eigenvalue $\lambda_{1}$ and build the rotation matrix $\mathbf{R}$ [Eq. (10)]. Finally, we rotate every particle of the instantaneous configuration using $\mathbf{R}$ [Eq. (26)].

Step IV. Associate each of the remaining particles in the reference with the particles in the instantaneous configuration using the following approach: find which particle $i$ in the instantaneous configuration is nearest particle $r$ in the reference; find which particle $r^{\prime}$ in the reference is closest to the particle $i$ found in the previous step; if $r \neq r^{\prime}$, then repeat the process with another particle $r$; or if $r=r^{\prime}$, then pair particles $i$ and $r$ and remove them from further consideration; continue the process until all particles $i$ and $r$ are paired.

It is convenient to define an integer array $M(N)$ to store the integer labels $i$ for the instantaneous configuration. Specifically, $M(1)$ stores the label that is paired with particle 1 of the reference, $M(2)$ stores the label that is paired with particle 2 of the reference, and so on.

Step $V$. Rotate the instantaneous configuration again using all the matched particles.

Here, we use the same procedure that we used in Step III, except that all the particles are used to construct the matrix $\mathbf{P}$.

The smart-darting algorithm for indistinguishable particles is similar to the algorithm for distinguishable particles given in Sec. II B. The principle difference between the indistinguishable and distinguishable algorithms is the replacement of $\mathcal{S}^{(1)}$ by $O^{(1)}$. Except for an additional modification in Step 4, the details of the two algorithms remain the same. In Step 4 in addition to checking if $\boldsymbol{\Gamma}^{\prime}$ is inside $\mathcal{S}^{(1)}$, we also test whether $\Gamma^{\prime}$ is in $O^{(1)}$. Specifically, we compare the integer arrays $M(r)$ and $M^{\prime}(r)$ belonging to $\boldsymbol{\Gamma}$ and $\boldsymbol{\Gamma}^{\prime}$, respectively. If $\boldsymbol{\Gamma}^{\prime}$ is in $O^{(1)}$, it must be true that 


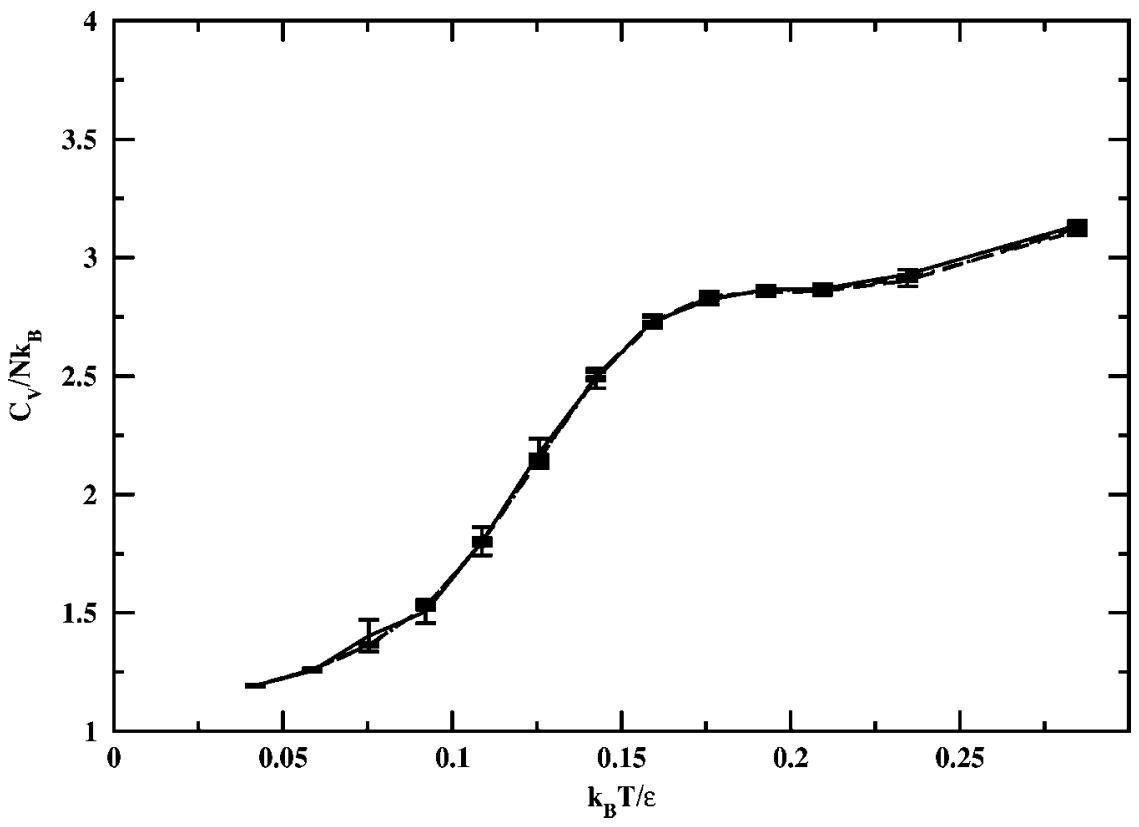

FIG. 1. The heat capacity per particle in units of $k_{B}$ as a function of temperature in units of $\epsilon / k_{B}$ for $\mathrm{LJ}_{7}$. The solid curve represents the data for the Metropolis calculation, the dashed curve represents data for smart darting, and the line with alternating dashes and dots represents data where smart darting is combined with parallel tempering. The error bars represent two standard deviations of the mean.

$M(r)=M^{\prime}(r) \forall r$.

To obtain $M^{\prime}(r)$, we apply Steps II-V to the new configuration as well.

\section{APPLICATION}

To illustrate the use of smart darting in Eckart space for a real physical system, we apply the approach to the calculation of the heat capacity of a seven-atom Lennard-Jones cluster. The system has been examined previously, ${ }^{26}$ and the heat capacity can be calculated accurately using standard Metropolis based approaches. Consequently, $\mathrm{LJ}_{7}$ provides a useful first investigation of the modified smart-darting method.

The seven-atom cluster is modeled using the standard Lennard-Jones interaction modified by an external constraining potential

$$
U\left(\left\{\mathbf{r}_{i}\right\}\right)=\sum_{i<j}^{N} u\left(r_{i j}\right)+\sum_{i=1}^{N} U_{c}\left(\mathbf{r}_{i}\right),
$$

where $r_{i j}$ is the distance between particles $i$ and $j$, and the constraining potential having radius $r_{c}$ is given by ${ }^{20}$

$$
U_{c}\left(\mathbf{r}_{i}\right)=\epsilon\left(\frac{\left|\mathbf{r}_{i}-\mathbf{r}_{c m}\right|}{r_{c}}\right)^{20} .
$$

In Eq. (41) $u$ is the Lennard-Jones potential,

$$
u(r)=4 \epsilon\left[\left(\frac{\sigma}{r}\right)^{12}-\left(\frac{\sigma}{r}\right)^{6}\right],
$$

with $\sigma$ and $\epsilon$ the usual length and energy parameters, and in Eq. (42) $\mathbf{r}_{c m}$ is the coordinate of the center of mass of the cluster. As has been discussed elsewhere, ${ }^{27} r_{c}$ must be chosen with some care. If $r_{c}$ is taken to be too small, the constraining potential can have significant effect on the thermodynamic properties within a phase change region. On the other hand, if $r_{c}$ is taken to be too large, evaporation events can make it difficult to attain ergodicity with any method. In this work, we take $r_{c}=1.68 \sigma$, a value that we have found by numerical experimentation gives the proper compromise between the two extremes.

All the calculations reported in this work consist of $10^{8}$ Monte Carlo points. The initial configuration has been chosen randomly, and $10^{6}$ parallel tempering points have been included in an equilibration step at each temperature prior to the accumulation of data. Both parallel tempering exchanges and smart-darting moves in Eckart space have been included with a frequency of $10 \%$.

A graph of the heat capacity per particle expressed in units of the Boltzmann constant $k_{B}$ as a function of temperature expressed in units of $\epsilon / k_{B}$ is shown in Fig. 1. The solid line represents the data obtained with the Metropolis method, the dashed line represents the smart darting results and the line with alternating dashes and dots represents the combined parallel tempering/smart darting results. The potential energy surface of $\mathrm{LJ}_{7}$ contains five potential minima, ${ }^{1}$ with the two minima highest in energy being chiral isomers that are equal in energy. All five potential energy minima are used as templates in the current smart-darting calculation with the lowest energy isomer used as the reference configuration. The rapid rise in the heat capacity at temperatures above $k_{B} T / \epsilon=0.05$ reflects isomerization transitions, and these isomerization transitions are often interpreted in terms of "cluster melting.," The melting region can be expected to be the most difficult temperature region to simulate, and such difficulties should be reflected by increased statistical errors in the computed quantities. Because it is difficult to resolve differences in Fig. 1, in Fig. 2 we display the statistical fluctuations of the heat capacity $2 \sigma$ (two standard deviations of the mean) as a function of the temperature using four methods. The line marked "Met" represents the Metropolis based methods, the line marked "sd" represents the data with pure smart darting, the line marked "pt" represents the parallel tempering data and the line marked "ptsd" represents the data where parallel tempering is combined with smart darting. Each calculation containing $10^{8}$ points with data accumulation has been run 


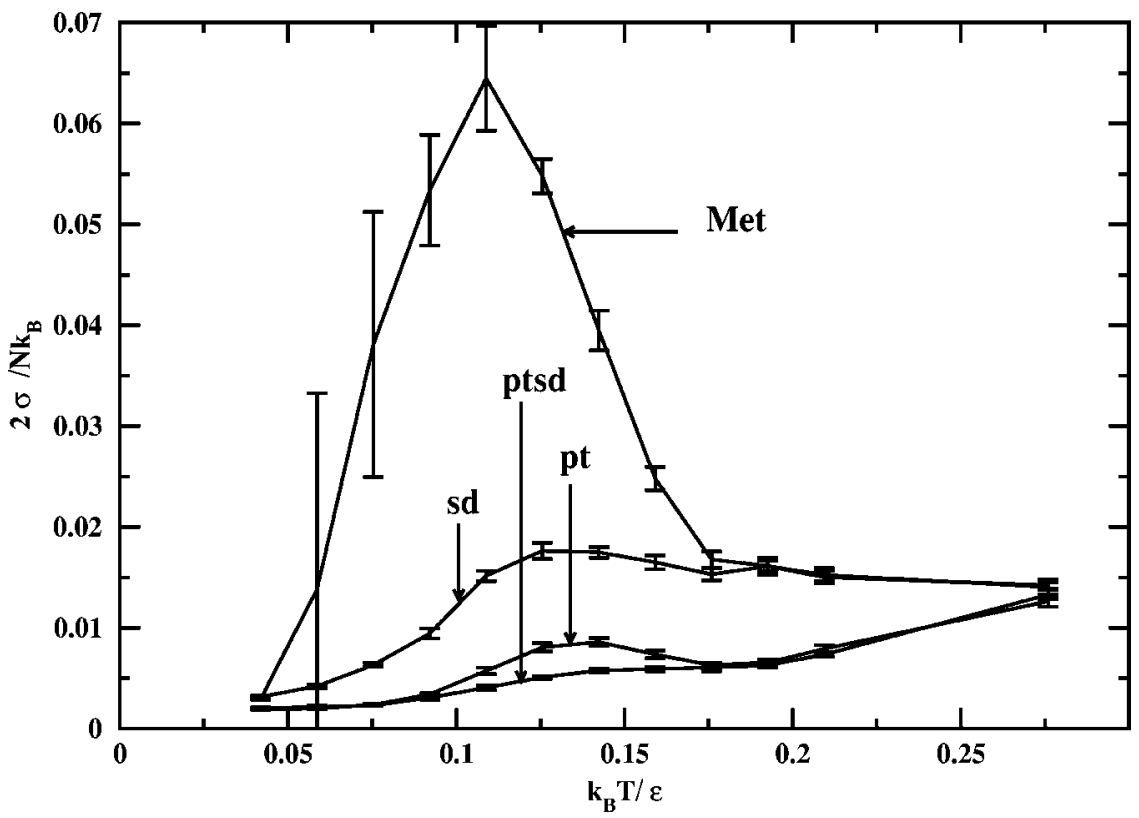

FIG. 2. Two standard deviations of the mean for the heat capacity per particle expressed in units of the Boltzmann constant as a function of the temperature expressed in units of $\epsilon / k_{B}$. The line marked Met represents the Metropolis data, the line marked sd represents the smart-darting data, the line marked pt represent the parallel tempering data, and the line marked ptsd represent the data when parallel tempering is combined with smart darting.

ten times with random initialization of the configurations, and the plotted points represent averages of $2 \sigma$ over the ten runs. The statistical fluctuations of the values of $2 \sigma$ obtained using each method are also included as error bars with the plotted data. With the inclusion of smart darting, there is a significant decrease in $2 \sigma$ above the temperatures where the isomerization transitions occur, and the decrease in $2 \sigma$ continues until the higher temperatures where the Metropolis method is expected to work well. The parallel tempering results clearly have smaller values of $2 \sigma$ than either pure Metropolis or smart darting. The best results are obtained by combining parallel tempering with smart darting, although smart darting improves parallel tempering only modestly compared to the improvements that pure parallel tempering or smart darting provide for the Metropolis results.

We can obtain further insight about the smart-darting method by examining the fraction $f$ of accepted smart- darting moves as a function of temperature. We display such data in Fig. 3. At low temperatures, the fraction of the smartdarting moves that are accepted is nearly zero. Prior to data collection, the initial configurations are thermally equilibrated, and at the lowest temperatures, the system executes small amplitude oscillatory motion about the lowest energy isomer of the system. At such temperatures, the probability of any isomerization event is small, and isomerization transitions are physically improbable. Consequently, only a small fraction of attempted darts is accepted at low temperatures. At temperatures where the heat capacity begins to rise with the associated isomerization transitions, we see an increase in the fraction of accepted smart-darting moves. It is interesting that only a small fraction of smart-darting moves needs to be accepted to observe a significant decrease in the variance of the heat capacity. In the region of temperature where the gap in $2 \sigma$ between the Metropolis and smart-

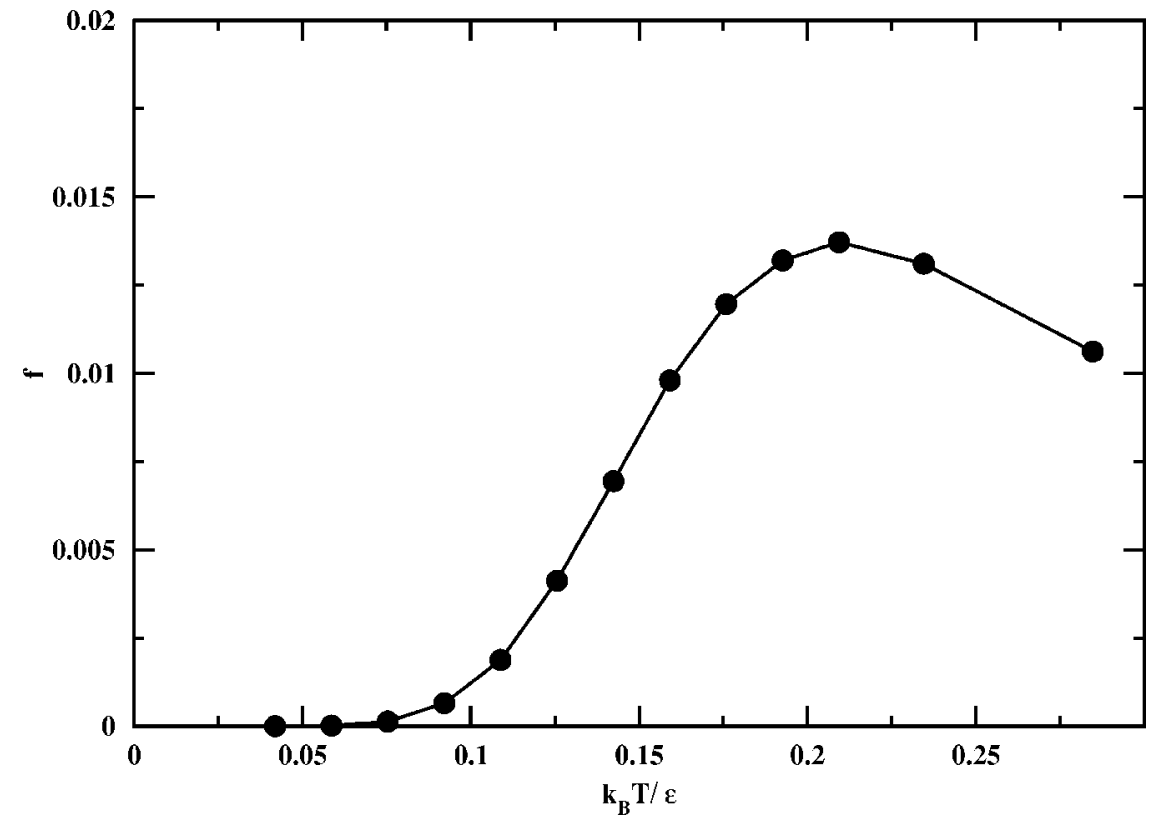

FIG. 3. The fraction of accepted smart-darting moves as a function of temperature expressed in units of $\epsilon / k_{B}$. 
darting results are greatest, the fraction of accepted moves is less than $1 \%$. Beyond temperatures where the heat capacity has reached a plateau and the gap in $2 \sigma$ between the Metropolis and smart-darting results is smallest, the fraction of accepted smart-darting moves reaches a maximum and begins a gradual decline.

\section{DISCUSSION}

In this work, we have augmented the smart-darting method so that the angular momentum constraints of the original algorithm ${ }^{7}$ can be removed. We have found the modifications required to remove the angular momentum constraints to be significant. The origin of the modifications is the reduced space used for the smart-darting moves. Using the formulation originally invented by Eckart, we have constructed an Eckart space for the required restricted smartdarting moves. Because of the special restrictions in the moves, we have found it necessary to include a Jacobian in the probability density.

In our application of the method to $\mathrm{LJ}_{7}$ we have found smart darting to improve significantly the statistical error of the calculated heat capacity when Metropolis methods are used. The improvements have been most pronounced in the isomerization (or melting) region of the heat capacity curve. It is in the melting region where the attainment of ergodicity can be most difficult. Smart darting also improves the statistical fluctuations of parallel tempering, but the improvement is less pronounced than the improvements found for Metropolis.

Unlike smart darting, parallel tempering calculations do not require the prior determination of any of the potential energy minima, and for the case studied here parallel tempering does a better job than smart darting alone. For clusters, we envision smart darting to be most useful as a method for augmenting parallel tempering. Future calculations can be expected for systems such as $\mathrm{LJ}_{38}$ or $\mathrm{LJ}_{75}$ where occasional smart-darting moves using a single dart vector between the two templates defined by the global minima in the two principal basins on the potential energy surface should allow ergodic simulations with decreased numbers of Monte Carlo points. We expect that this procedure can make systems like $\mathrm{LJ}_{75}$ tractable.

\section{ACKNOWLEDGMENTS}

This work was supported in part by National Science Foundation Grant Nos. CHE-0095053 and CHE-0131114.

\section{APPENDIX: THE JACOBIAN}

In this appendix, we discuss our choice of internal coordinates and the Jacobian of the tranformation into generalized coordinates.

\section{The internal coordinates}

The internal coordinates are the $(3 N-6)$ coordinates that span Eckart space. To describe a configuration, we start with $3 N$ Cartesian coordinates referred to the center of mass. Of those coordinates, three are already redundant because of the center of mass condition [Eq. (1)]. The Eckart condition [Eq. (2)] introduces three linear relations that makes three additional coordinates redundant. We then obtain $(3 N-6)$ independent, Cartesian coordinates to specify a configuration. We take those independent, Cartesian coordinates to be the internal coordinates. It is convenient to rewrite the Eckart condition,

$$
\sum_{i=1}^{N-1} \widetilde{\mathbf{s}}_{i} \times \mathbf{r}_{i}=0,
$$

where

$$
\widetilde{\mathbf{s}}_{i}=\widetilde{\mathbf{r}}_{i}-\sqrt{\frac{m_{i}}{m_{N}}} \widetilde{\mathbf{r}}_{N} .
$$

To obtain Eq. (A1), we have made use of Eq. (1) to eliminate three redundant, Cartesian coordinates.

To find a suitable set of $(3 N-6)$ independent coordinates, we express three of the coordinates as functions of the remaining ones. The choice of these three coordinates is not arbitrary in that certain choices are unsuitable. For example, if we consider the set $\left\{x_{N-1}, y_{N-1}, z_{N-1}\right\}$, and expand Eq. (A1), we obtain

$$
\begin{aligned}
& y_{N-1} c_{N-1}-z_{N-1} b_{N-1}=\sum_{i=1}^{N-2}\left(z_{i} b_{i}-y_{i} c_{i}\right), \\
& x_{N-1} c_{N-1}-z_{N-1} a_{N-1}=\sum_{i=1}^{N-2}\left(z_{i} a_{i}-x_{i} c_{i}\right), \\
& x_{N-1} b_{N-1}-y_{N-1} a_{N-1}=\sum_{i=1}^{N-2}\left(y_{i} a_{i}-x_{i} b_{i}\right),
\end{aligned}
$$

where $\widetilde{\mathbf{s}}_{i} \equiv\left(a_{i}, b_{i}, c_{i}\right)$. In this example, the determinant of coeficients is identically zero:

$$
\operatorname{det}\left[\begin{array}{ccc}
0 & c_{N-1} & -b_{N-1} \\
c_{N-1} & 0 & -a_{N-1} \\
b_{N-1} & -a_{N-1} & 0
\end{array}\right]=0 .
$$

Consequently, the coordinates of the set $\left\{x_{N-1}, y_{N-1}, z_{N-1}\right\}$ cannot be expressed as functions of the $(3 N-6)$ other coordinates. The same situation arises for the sets $\left\{x_{i}, y_{i}, z_{i}\right\}$, $\left\{x_{i}, x_{j}, x_{k}\right\},\left\{y_{i}, y_{j}, y_{k}\right\}$, and $\left\{z_{i}, z_{j}, z_{k}\right\}$. A proper set of $(3 N-6)$ independent, Cartesian coordinates must avoid this inconsistency.

\section{The Jacobian}

Given an inertial frame of reference, fixed at the center of mass of a configuration, only $(3 N-3)$ Cartesian coordinates are required to describe the configuration. The classical canonical probability of finding a configuration with energy $U$ is

$$
p=\frac{e^{-\beta U}}{z(\beta)} d \mathbf{r}_{1} d \mathbf{r}_{2} \cdots d \mathbf{r}_{N-1},
$$

where 


$$
z(\beta)=\int e^{-\beta U} d \mathbf{r}_{1} d \mathbf{r}_{2} \cdots d \mathbf{r}_{N-1} .
$$

The present work makes it necessary to express $p$ as a function of a convenient set of generalized coordinates. These coordinates are the three Euler angles $\left\{\theta_{i}\right\}$ and the $(3 N-6)$ internal, Cartesian coordinates $\left\{q_{i}\right\}$. The probability $p$ in Eq. (A5) is now written as

$$
p=\frac{e^{-\beta U}}{z(\beta)} J\left(\left\{\theta_{i}\right\},\left\{q_{j}\right\}\right) d \theta_{1} d \theta_{2} d \theta_{3} d q_{1} d q_{2} \cdots d q_{3 N-6},
$$

where $J\left(\left\{\theta_{i}\right\},\left\{q_{j}\right\}\right)$ is the Jacobian of transformation into the new coordinates.
To determine $J\left(\left\{\theta_{i}\right\},\left\{q_{j}\right\}\right)$ for a system with $N \geqslant 3$, we consider the transformation equations

$$
\mathbf{r}_{i}^{I}=\mathbf{R}^{T}\left(\theta_{j}\right) \mathbf{r}_{i}\left(q_{k}\right) i=1, \ldots, N-1,
$$

where the position vectors $\mathbf{r}_{i}^{I}$ and $\mathbf{r}_{i}$ are referred to the inertial frame and the Eckart frame respectively. In terms of Euler angles, the rotation matrix is ${ }^{22}$

$$
\mathbf{R}^{T}=\left[\begin{array}{lll}
\cos \theta_{1} \cos \theta_{3}-\sin \theta_{1} \cos \theta_{2} \sin \theta_{3} & -\cos \theta_{1} \sin \theta_{3}-\sin \theta_{1} \cos \theta_{2} \cos \theta_{3} & \sin \theta_{1} \cos \theta_{2} \\
\sin \theta_{1} \cos \theta_{3}+\cos \theta_{1} \cos \theta_{2} \sin \theta_{3} & -\sin \theta_{1} \sin \theta_{3}+\cos \theta_{1} \cos \theta_{2} \cos \theta_{3} & -\cos \theta_{1} \sin \theta_{2} \\
\sin \theta_{2} \sin \theta_{3} & \sin \theta_{2} \cos \theta_{3} & \cos \theta_{2}
\end{array}\right] .
$$

From the discussion in preceding section, we choose the set $\left\{z_{N-2}, y_{N-1}, z_{N-1}\right\}$ to be the redundant coordinates (in the Eckart frame) so that the transformation is

$$
\left\{\mathbf{r}_{1}^{I}, \ldots, \mathbf{r}_{N-1}^{I}\right\} \rightarrow\left\{\theta_{1}, \theta_{2}, \theta_{3}, \mathbf{r}_{1}, \ldots, \mathbf{r}_{N-3}, x_{N-2}, y_{N-2}, x_{N-1}\right\} .
$$

The Jacobian determinant of this transformation takes on the form

$$
J=\operatorname{det}\left[\begin{array}{ccccccc}
\mathbf{Y}_{1} & \mathbf{R}^{T} & \mathbf{0} & \mathbf{0} & \cdots & \mathbf{0} & \mathbf{0} \\
\mathbf{Y}_{2} & \mathbf{0} & \mathbf{R}^{T} & \mathbf{0} & \cdots & \mathbf{0} & \mathbf{0} \\
\mathbf{Y}_{3} & \mathbf{0} & \mathbf{0} & \mathbf{R}^{T} & \cdots & \mathbf{0} & \mathbf{0} \\
\vdots & \vdots & \vdots & \vdots & \ddots & \vdots & \vdots \\
\mathbf{Y}_{N-3} & \mathbf{0} & \mathbf{0} & \mathbf{0} & \cdots & \mathbf{R}^{T} & \mathbf{0} \\
\mathbf{Y}_{N-2} & \mathbf{H}_{1} & \mathbf{H}_{2} & \mathbf{H}_{3} & \cdots & \mathbf{H}_{N-3} & \mathbf{H}_{0} \\
\mathbf{Y}_{N-1} & \mathbf{G}_{1} & \mathbf{G}_{2} & \mathbf{G}_{3} & \cdots & \mathbf{G}_{N-3} & \mathbf{G}_{0}
\end{array}\right]
$$

where the nonzero $3 \times 3$ blocks are defined, in column-vector notation, by

$$
\begin{aligned}
& \mathbf{Y}_{i}=\left[\begin{array}{lll}
\frac{\partial x_{i}^{I}}{\partial \theta_{1}} & \frac{\partial x_{i}^{I}}{\partial \theta_{2}} & \frac{\partial x_{i}^{I}}{\partial \theta_{3}} \\
\frac{\partial y_{i}^{I}}{\partial \theta_{1}} & \frac{\partial y_{i}^{I}}{\partial \theta_{2}} & \frac{\partial y_{i}^{I}}{\partial \theta_{3}} \\
\frac{\partial z_{i}^{I}}{\partial \theta_{1}} & \frac{\partial z_{i}^{I}}{\partial \theta_{2}} & \frac{\partial z_{i}^{I}}{\partial \theta_{3}}
\end{array}\right] \\
& \equiv\left[\frac{\partial \mathbf{r}_{i}^{I}}{\partial \theta_{1}} \frac{\partial \mathbf{r}_{i}^{I}}{\partial \theta_{2}} \frac{\partial \mathbf{r}_{i}^{I}}{\partial \theta_{3}}\right] i=1, \ldots, N-1, \\
& \mathbf{H}_{i}=\left[\frac{\partial \mathbf{r}_{N-2}^{I}}{\partial x_{i}} \frac{\partial \mathbf{r}_{N-2}^{I}}{\partial y_{i}} \frac{\partial \mathbf{r}_{N-2}^{I}}{\partial z_{i}}\right] i=1, \ldots, N-3,
\end{aligned}
$$

$$
\begin{aligned}
\mathbf{G}_{i} & =\left[\frac{\partial \mathbf{r}_{N-1}^{I}}{\partial x_{i}} \frac{\partial \mathbf{r}_{N-1}^{I}}{\partial y_{i}} \frac{\partial \mathbf{r}_{N-1}^{I}}{\partial z_{i}}\right] i=1, \ldots, N-3, \\
\mathbf{H}_{0} & =\left[\frac{\partial \mathbf{r}_{N-2}^{I}}{\partial x_{N-2}} \frac{\partial \mathbf{r}_{N-2}^{I}}{\partial y_{N-2}} \frac{\partial \mathbf{r}_{N-2}^{I}}{\partial x_{N-1}}\right],
\end{aligned}
$$

and

$$
\mathbf{G}_{0}=\left[\frac{\partial \mathbf{r}_{N-1}^{I}}{\partial x_{N-2}} \frac{\partial \mathbf{r}_{N-1}^{I}}{\partial y_{N-2}} \frac{\partial \mathbf{r}_{N-1}^{I}}{\partial x_{N-1}}\right] \text {. }
$$

An useful theorem about partitioned matrices ${ }^{28}$ says that, for a square matrix $\mathbf{M}$ partitioned as

$$
\mathbf{M}=\left[\begin{array}{ll}
\mathbf{A} & \mathbf{B} \\
\mathbf{C} & \mathbf{D}
\end{array}\right],
$$

with $\mathbf{A}$ and $\mathbf{D}$ being $m \times m$ and $n \times n$, respectively, the determinant of $\mathbf{M}$ is given by

$$
\operatorname{det} \mathbf{M}=\operatorname{det} \mathbf{A} \operatorname{det}\left(\mathbf{D}-\mathbf{C A}^{-1} \mathbf{B}\right),
$$

provided that $\mathbf{A}$ is invertible. To apply that theorem to Eq. (A11), we rearrange and partition the matrix in Eq. (A11),

$$
J=\operatorname{det}\left[\begin{array}{lllll|ll}
\mathbf{R}^{T} & \mathbf{0} & \mathbf{0} & \cdots & \mathbf{0} & \mathbf{0} & \mathbf{Y}_{1} \\
\mathbf{0} & \mathbf{R}^{T} & \mathbf{0} & \cdots & \mathbf{0} & \mathbf{0} & \mathbf{Y}_{2} \\
\mathbf{0} & \mathbf{0} & \mathbf{R}^{T} & \cdots & \mathbf{0} & \mathbf{0} & \mathbf{Y}_{3} \\
\vdots & \vdots & \vdots & \ddots & \vdots & \vdots & \vdots \\
\mathbf{0} & \mathbf{0} & \mathbf{0} & \cdots & \mathbf{R}^{T} & \mathbf{0} & \mathbf{Y}_{N-3} \\
\hline \mathbf{H}_{1} & \mathbf{H}_{2} & \mathbf{H}_{3} & \cdots & \mathbf{H}_{N-3} & \mathbf{H}_{0} & \mathbf{Y}_{N-2} \\
\mathbf{G}_{1} & \mathbf{G}_{2} & \mathbf{G}_{3} & \cdots & \mathbf{G}_{N-3} & \mathbf{G}_{0} & \mathbf{Y}_{N-1}
\end{array}\right] .
$$

Using Eq. (A19), we transform Eq. (A20) into 


$$
J=\operatorname{det}\left[\begin{array}{cc}
\mathbf{H}_{0} & \left(\mathbf{Y}_{N-2}-\sum_{i=1}^{N-3} \mathbf{H}_{i} \mathbf{R} \mathbf{Y}_{i}\right) \\
\mathbf{G}_{0} & \left(\mathbf{Y}_{N-1}-\sum_{i=1}^{N-3} \mathbf{G}_{i} \mathbf{R} \mathbf{Y}_{i}\right)
\end{array}\right] .
$$

The Jacobian $J$ of Eq. (A21) is a $6 \times 6$ determinant and, unfortunately, it cannot be reduced further using Eq. (A19); none of its blocks is invertible. Except for $N=3$, direct expansion is hindered by an overwhelming number of terms. For example, if $M$ represents the largest number of terms in one of the 36 elements of Eq. (A21), then an upper limit to the total number of terms after the expansion is $(6 \times 6 !) M$, which amounts to 21600 for $M=5$. To circumvent that problem, we first express $J$ as a determinant of 6, 6-dimensional column vectors:

$$
\begin{aligned}
J= & \operatorname{det}\left[\mathbf { a } _ { 1 } \mathbf { a } _ { 2 } \mathbf { a } _ { 3 } ( \mathbf { a } _ { 4 } - \sum _ { i = 1 } ^ { N - 3 } \mathbf { b } _ { i } ) ( \mathbf { a } _ { 5 } - \sum _ { i = 1 } ^ { N - 3 } \mathbf { c } _ { i } ) \left(\mathbf{a}_{6}\right.\right. \\
& \left.\left.-\sum_{i=1}^{N-3} \mathbf{d}_{i}\right)\right]
\end{aligned}
$$

where $\mathbf{a}_{1}$ is the left most column vector in Eq. (A21), $\mathbf{a}_{2}$ is the next six-dimensional column vector in Eq. (A21), and so on. We then use the multilinearity property of determinants ${ }^{25}$ to write

$$
J=\sum_{j=0}^{N-3} \sum_{k=0}^{N-3} \sum_{l=0}^{N-3} \operatorname{det}\left[\mathbf{a}_{1} \mathbf{a}_{2} \mathbf{a}_{3} \boldsymbol{\alpha}_{j} \boldsymbol{\beta}_{k} \boldsymbol{\gamma}_{l}\right]
$$

where

$$
\begin{array}{ll}
\boldsymbol{\alpha}_{0}=\mathbf{a}_{4}, & \boldsymbol{\alpha}_{i}=-\mathbf{b}_{i}, \\
\boldsymbol{\beta}_{0}=\mathbf{a}_{5}, & \boldsymbol{\beta}_{i}=-\mathbf{c}_{i} ;
\end{array}
$$

and

$$
\boldsymbol{\gamma}_{0}=\mathbf{a}_{6}, \quad \boldsymbol{\gamma}_{i}=-\mathbf{d}_{i} .
$$

The determinants in Eq. (A23) become tractable by using Mathematica. ${ }^{29}$ The total number of terms in the sum is $(N$ $-2)^{3}$. Expanding the sum for the first few values of $N$, we find that $J$ can be recast to yield the expression

$$
J=\sin \theta_{2}\left|\frac{\bar{J}}{c}\right|,
$$

where $c$ is the determinant of coefficients of the set $\left\{z_{N-2}, y_{N-1}, z_{N-1}\right\}$. The function $\bar{J}$ is the part of the Jacobian depending only on the internal coordinates and is defined by

$$
\begin{aligned}
\bar{J}= & \sum_{i=1}^{N-2} \sum_{j=i+1}^{N-1} \nu_{i, j}\left(\mu_{i}+\mu_{j}\right)+\sum_{i=1}^{N-3} \sum_{j=i+1}^{N-2} \sum_{k=j+1}^{N-1}\left(\nu_{i, j} \mu_{k}+\nu_{j, k} \mu_{i}\right. \\
& \left.+\nu_{k, i} \mu_{j}-\sigma_{i, j, k}\right),
\end{aligned}
$$

with

$$
\begin{aligned}
& \mu_{i}=\widetilde{\mathbf{s}}_{i} \cdot \mathbf{r}_{i}, \\
& \nu_{i, j}=\left(\widetilde{\mathbf{s}}_{i} \times \widetilde{\mathbf{s}}_{j}\right) \cdot\left(\mathbf{r}_{i} \times \mathbf{r}_{j}\right),
\end{aligned}
$$

and

$$
\sigma_{i, j, k}=\left[\widetilde{\mathbf{s}}_{i} \cdot\left(\widetilde{\mathbf{s}}_{j} \times \widetilde{\mathbf{s}}_{k}\right)\right]\left[\mathbf{r}_{i} \cdot\left(\mathbf{r}_{j} \times \mathbf{r}_{k}\right)\right] .
$$

The vectors $\widetilde{\mathbf{s}}_{i}$ have been defined in Eq. (A2).

${ }^{1}$ D. L. Freeman and J. D. Doll, Annu. Rev. Phys. Chem. 47, 43 (1996).

${ }^{2}$ R. S. Berry, T. L. Beck, and H. Davis, Adv. Chem. Phys. 70, 75 (1988).

${ }^{3}$ D. D. Frantz, D. L. Freeman, and J. Doll, J. Chem. Phys. 93, 2769 (1990).

${ }^{4}$ E. Marinari and G. Parisi, Europhys. Lett. 19, 451 (1992).

${ }^{5}$ C. J. Geyer and E. A. Thompson, J. Am. Stat. Assoc. 90, 909 (1995).

${ }^{6}$ M. Falcioni and M. Deem, J. Chem. Phys. 110, 1754 (1999).

${ }^{7}$ I. Andricioaei, J. E. Straub, and A. F. Voter, J. Chem. Phys. 114, 6994 (2001).

${ }^{8}$ I. Andricioaei and J. E. Straub, J. Chem. Phys. 107, 9117 (1997).

${ }^{9}$ J. P. Neirotti, F. Calvo, D. L. Freeman, and J. D. Doll, J. Chem. Phys. 112, 10340 (2000).

${ }^{10}$ F. Calvo, J. P. Neirotti, D. L. Freeman, and J. D. Doll, J. Chem. Phys. 112, 10350 (2000).

${ }^{11}$ J. P. K. Doye, M. A. Miller, and D. J. Wales, J. Chem. Phys. 110, 6896 (1999).

${ }^{12}$ L. J. Munro, A. Tharrington, and K. D. Jordan, Comput. Phys. Commun. 145, 1 (2002).

${ }^{13}$ D. Sabo, C. Predescu, J. D. Doll, and D. L. Freeman, J. Chem. Phys. 121, 856 (2004).

${ }^{14}$ J. P. K. Doye, M. A. Miller, and D. J. Wales, J. Chem. Phys. 111, 8417 (1999).

${ }^{15}$ H. Xu and B. J. Berne, J. Chem. Phys. 110, 10299 (1999).

${ }^{16}$ F. Calvo and J. P. K. Doye, Phys. Rev. E 63, 010902(R) (2001).

${ }^{17}$ R. Zhou and B. J. Berne, J. Chem. Phys. 107, 9185 (1997).

${ }^{18}$ A. F. Voter, J. Chem. Phys. 82, 1890 (1985).

${ }^{19}$ N. Metropolis, A. W. Rosenbluth, M. N. Rosenbluth, A. H. Teller, and E. Teller, J. Chem. Phys. 21, 1087 (1953).

${ }^{20}$ C. Eckart, Phys. Rev. 47, 552 (1935).

${ }^{21}$ J. D. Louck and H. W. Galbraith, Rev. Mod. Phys. 48, 69 (1976).

${ }^{22}$ H. Goldstein, Classical Mechanics (Addison-Wesley, Reading, MA, 1980).

${ }^{23}$ E. B. Wilson, J. C. Decius, and P. C. Cross, Molecular Vibrations (Dover, New York, 1980).

${ }^{24}$ S. Califano, Vibrational States (Wiley, New York, 1976).

${ }^{25}$ T. M. Apostol, Calculus (Wiley, New York, 1969).

${ }^{26}$ D. D. Frantz, D. L. Freeman, and J. D. Doll, J. Chem. Phys. 97, 5713 (1992).

${ }^{27}$ J. P. Neirotti, D. L. Freeman, and J. D. Doll, Phys. Rev. E 62, 7445 (2000).

${ }^{28}$ F. Zhang, Matrix Theory: Basic Results and Thechniques (Springer, New York, 1999).

${ }^{29}$ S. Wolfram, The Mathematica Book (Cambridge University Press, New York, 1999). 
The Journal of Chemical Physics is copyrighted by the American Institute of Physics (AIP). Redistribution of journal material is subject to the AIP online journal license and/or AIP copyright. For more information, see http://ojps.aip.org/jcpo/jcpcr/jsp 